\title{
Designing Expressive Lights and In-Situ Motions for Robots to Express Emotions
}

\author{
Sichao Song \\ Department of Informatics, the Graduate University for \\ Advanced Studies (SOKENDAI) \\ Tokyo, Japan \\ sichaos@nii.ac.jp
}

\author{
Seiji Yamada \\ National Institute of Informatics \\ Tokyo, Japan \\ seiji@nii.ac.jp
}

\begin{abstract}
In this paper, we explore how a utility robot might express emotions via expressive lights and in-situ motions. In most previous work, methods for either modality were investigated alone, leaving a huge potential to improve the expression of emotions by combining the two modalities. We present a series of three studies, one for investigating how well people might recognize emotions on the basis of expressive light cues alone, one for exploring how people might perceive affect towards in-situ motion characteristics, and one for further combining the two modalities and studying whether multimodal expressions could be better recognized by people. Results from the first study show participants were not able to recognize target emotions with high accuracy. Results from the second suggest a relationship between the in-situ motion characteristics of a robot and perceived affect. Results from the third suggest that expressions that combine in-situ motions with expressive lights were better able to convey many emotions but not all. We conclude that adding in-situ motions to affective expressive lights appears to be better able to help convey emotions. These findings are important for designing affective behaviors for future utility robots that need to possess certain social abilities.
\end{abstract}

\section{KEYWORDS}

Expressive lights, appearance-constrained robot, in-situ motion, affective computing, emotion, human-robot interaction (HRI)

\section{ACM Reference Format:}

Sichao Song and Seiji Yamada. 2018. Designing Expressive Lights and In-Situ Motions for Robots to Express Emotions. In 6th International Conference on Human-Agent Interaction (HAI '18), December 15-18, 2018, Southampton, United Kingdom. ACM, New York, NY, USA, 7 pages. https://doi.org/10.1145/ 3284432.3284458

\section{INTRODUCTION}

There is an increasing need for utility robots to express emotions. People often expect such robots to act socially [1]. For instance, in previous work, it was found that rescue workers expect a small tanklike robot to follow social conventions [2]; man-packable robots

Permission to make digital or hard copies of all or part of this work for personal or classroom use is granted without fee provided that copies are not made or distributed for profit or commercial advantage and that copies bear this notice and the full citation on the first page. Copyrights for components of this work owned by others than ACM must be honored. Abstracting with credit is permitted. To copy otherwise, or republish, to post on servers or to redistribute to lists, requires prior specific permission and/or a fee. Request permissions from permissions@acm.org.

HAI '18, December 15-18, 2018, Southampton, United Kingdom

(C) 2018 Association for Computing Machinery.

ACM ISBN 978-1-4503-5953-5/18/12 . \$15.00

https://doi.org/10.1145/3284432.3284458 were perceived as "creepy" and not reassuring when they were operated close to simulated victims [3]. However, because utility robots are, in general, restricted in appearance, there is a lack of methods that can be used for these robots to express affect and intent.

Due to the restricted interaction methods available to utility robots, human-robot interaction (HRI) approaches rely mainly on motion cues [4-7]. Strong relationships between motion parameters, e.g., acceleration, curvature, and trajectory, were found, and the type of body that a robot has did not seem to affect such relationships [4]. Nevertheless, motion alone was not able to convey emotions precisely, although motion parameters might be used to predict the perceived arousal and valence. Moreover, current methods regarding robot motion can be hard to apply in many practical scenarios. For instance, it can be impossible for a robot to use big movements to interact with people when situated in a narrow corridor or a crowded room. In addition, making big movements takes a rather long time, which would likely result in users becoming frustrated as it would take a long time to understand what a robot is expressing.

In addition to motion, using expressive lights as dynamic visual cues has been explored for designing affective HRI as well $[8,9]$. Researchers found that a robot is able to convey emotions by showing expressive lights that dynamically change in color luminosity [8]. Parameters such as color, period, and waveform contribute to the perception of emotions. In particular, color is considered to be a strong cue for predicting perceived affect. Color psychologists have intensively investigated various aspects of color, including the effects of color on psychological and biological functioning [10]. However, using expressive lights alone did not increase the recognition accuracy as well, although doing so seemed to lead to better performance than using robot motion alone. In addition, approaches using expressive lights are inappropriate if a user has a color-vision disorder.

\subsection{Contribution}

In this work, we explore whether multi-model expressions that combine motion and expressive light cues might better convey target emotions. Particularly, we investigate and apply in-situ motions, rather than the motion patterns studied in previous work, in the hope of allowing a robot to express affect in a rather short time frame without making big movements. Our first experiment was performed to evaluate how well a robot might convey emotions on the basis of expressive lights alone. The design of the lights was adapted from previous work [8]. A second experiment was performed to explore how people might perceive affect towards in-situ 
motions since this was not clear due to lack of related literature. On the basis of the results of the two studies, a third experiment was performed to further investigate whether combining motion and expressive lights modalities might better convey emotions. In this paper, we use the Circumplex Model [11], a two-dimensional space, to investigate the design space. On the basis of participants' ratings of perceived emotions using the Self-Assessment Mannequin (SAM) method [12], we found that adding in-situ motions to expressive light cues helped with more precisely expressing emotions for happiness, sadness, disgust, and surprise. With this work, we hope to suggest an effective method for evaluating and comparing the emotional responses of users to robot expressions via different modalities and to further provide insights into designing affective expressions for utility robots.

\section{RELATED WORK}

Emotional expressions based on motion cues have been investigated in many studies. Tremoulet and Feldman [7] demonstrated that even a single moving object can be perceived as alive. They discovered that people's ratings of animacy were heavily influenced by the changes in speed and direction of an object. Particularly, in HRI, Saerbeck and Bartneck [4] explored the relationship between the motion features of a robot and the attribution of affect. They found a strong relationship between motion parameters and perceived affect, while the type of body that a robot has had no effect. Specifically, their results indicated that the level of acceleration can be used to predict the perceived arousal and that an interaction effect between acceleration and curvature existed with regards to valence information. Syrdal et al. [17] performed a video humanrobot interaction study in which participants viewed a video in which an appearance-constrained robot used dog-inspired affective cues to communicate affinity. They suggested that such cues be effective for non-verbal affective communication. Cauchard et al. [6] explored how personality traits and emotional attributes can be encoded in drones via their flight paths. They found that drone movements, such as speed, altitude, and orientation, were important for designing affective expressions.

Expressive lights used as dynamic visual cues have also been explored for HRI research and applications. For instance, both Sony's robot dog AIBO and Aldebaran's Nao use LED lights to assist in affective expression. Terada et al. [8] studied how a robot might convey emotions by dynamically changing the color luminosity of its body. Their findings suggest a relationship between hue value and basic types of emotion and that duration and waveform represent the intensity of emotion. Rea et al. [16] mounted multi-color LEDs on an iRobot Roomba robot to broadcast ambient information in the form of a colored halo. They investigated how a robot with an ambient light display may integrate into a daily environment. Song and Yamada [9] evaluated the effects of three modalities, color, sound, and vibration, on a human's emotional perception of a simple-shaped robot. Their results indicate that color can be an important cue for people to recognize emotions.

Besides, few studies explored design for multi-modal emotion expression for social robots in which both color and motion cues were employed. Häring et al. [18] designed eight expressions for a Nao robot for the emotions anger, sadness, fear, and joy, consisting of body movements, Sounds and Eye Colors. Their analysis suggested that body movements were appropriate for their target emotion but colors were unreliable. Löffler et al. [19] built a simple movable robot and designed a set of 28 uni- and multi- modal expressions for conveying four basic emotions joy, sadness, fear, and anger. They found that planar motions were the most effective uni-modal expressions but multi-modal expressions that used both color and motion offered overall best performance.

\section{METHODOLOGY}

\subsection{Emotional Model}

Among a number of proposed psychological models for the cognitive structure of emotions, two of them are widely accepted and supported by empirical evidence. Ekman and Friesen [13] suggested a set of basic emotions based on human facial expressions. Their set consists of anger, disgust, fear, joy, sadness, and surprise. Others evaluated facial expressions for the mental states of boredom, confusion, happiness, interest, and surprise [14] or anger, fear, happiness, sadness, and surprise [15]. To not view emotions as categories, Russell [11] introduced the Circumplex Model of Affect, in which emotions are mapped to a two-dimensional space: the arousal of an emotion and the valence of an experience. In previous studies [5] in which this emotional model was applied, it was found that the model was useful with regards to designing affective expressions, and it was suggested that it a more precise tool for evaluating the accuracy of emotion perception.

\subsection{Robot Configuration}

We used an iRobot Create 2 robot. Roomba is a series of autonomous robotic vacuum cleaners used in indoor environments. All Roomba robots are disc-shaped, $34 \mathrm{~cm}$ in diameter, and less than $9 \mathrm{~cm}$ in height. This robot perfectly fits the definition of a utility robot and, due to its constrained appearance, has very limited ways of expressing affect, e.g., moving forward/backward and spinning.

Figure 1 shows the configuration of the Roomba robot with an LED lighting system installed on it. We used one meter of a NeoPixel LED strip (60 pixels). The LED strip was controlled by an Arduino Uno R3 board, and both the strip and board were powered by a $5-\mathrm{V}, 3-\mathrm{A}$ portable power-bank. The same board was also used to control the movements of the robot. iRobot Create 2 robot provides

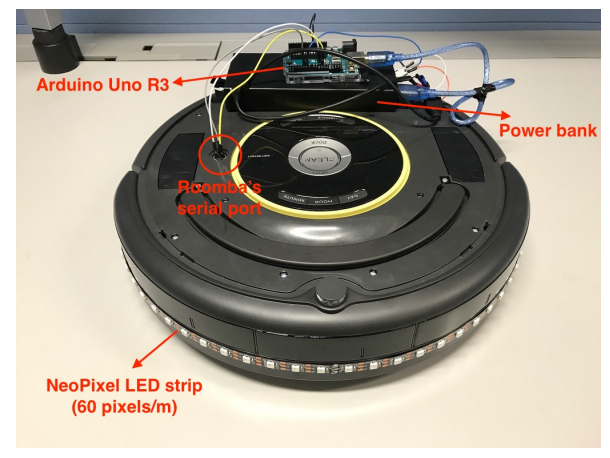

Figure 1: Configuration of Roomba robot with LED lighting system. 
the Roomba Open Interface (OI), which is a software interface for controlling and manipulating Roomba's behavior.

\subsection{Procedure}

In this work, we evaluated the effectiveness of expressions for seven emotions: anger, surprise, disgust, sadness, happiness, fear, and calm. These emotions were chosen on the basis of a model by Ekman and Friesen [13] and from similar studies [8]. To investigate how well these emotions might be recognized, we used the Circumplex Model. Basically, all seven emotions are featured in the model, covering the whole two-dimensional space (a broad spectrum of valence and arousal levels). For evaluation, we applied the SAM method. Participants rated emotions on a five-point valence scale (from very negative to very positive) as well as a five-point arousal scale (from very low to very high). The validity of using SAM scales to rate perceived emotions has already been shown in previous work [5, 12].

We performed three studies, where we designed the third study on the basis of the findings from the first two. Particularly, in study I, we asked participants to rate the seven emotions by using the SAM scales. This procedure was also taken by Strohmeier et al. [5] to account for potential individual variance in the interpretation of emotions. We used the results from this test as referential ratings of the seven emotions and to further obtain distance measures between the reference points and other ratings of perceived emotions. To be specific, with regards to each emotion, we obtained a referential $(X, Y)$ coordinate on the Circumplex Model. For further evaluations, e.g., emotional perception towards expressions based on expressive lights alone and expressions that combined in-situ motions and expressive lights, we then calculated the Euclidian distance between the response emotion and the references. Note that all distances were measured on a five-point SAM scale.

\subsection{Hypothesis}

We hypothesized that emotional expressions based on expressive lights alone would not able to be recognized with high accuracy. This would be revealed in study I by obtaining distance measures between ratings of such expressions and corresponding referential ratings. We also hypothesized that a strong relationship between in-situ motion parameters, e.g., speed and pattern, and participants' ratings of perceived emotions would be observed. This would be evaluated in study 2. Further, we hypothesized that multi-modal expressions that appropriately combine in-situ motions and expressive lights were better able to be recognized. Study III would test such a hypothesis.

\section{STUDY I: EMOTIONAL EXPRESSION VIA EXPRESSIVE LIGHTS}

In this study, we evaluated how well our Roomba robot might convey emotions on the basis of expressive lights alone. We also asked participants to rate the seven emotions by using the SAM scales, where the results were used as referential ratings of the emotions in both studies I and III.
Table 1: Parameter settings for design of expressive light expressions.

\begin{tabular}{c|ccc}
\hline Emotion & RGB & Period $[\mathrm{ms}]$ & Waveform \\
\hline Anger & $255,17,0$ & 896 & square \\
Surprise & $255,132,0$ & 747 & square \\
Disgust & $255,0,179$ & 1645 & square \\
Sadness & $21,0,255$ & 3310 & sinusoid \\
Happiness & $255,157,0$ & 1123 & square \\
Fear & $166,0,255$ & 1377 & square \\
Calm & $255,255,255$ & 2000 & sinusoid \\
\hline
\end{tabular}

\subsection{Method}

Our design of expressive lights was mainly adapted from Terada et al. [8]. Table 1 demonstrates the parameter settings used in our experiment. Note that we converted their hue values to RGB values to fit our LED lighting system. In addition, we also converted their mixed waveforms by simply applying the following rules; if the mix ratio is less than 0.5 , use a square waveform; if the mix ratio is greater than 0.5 , use a sinusoidal waveform.

\subsection{Participants}

Eighteen Japanese in total (12 males and 6 females) ranging from 22 to 50 years old $(M=29.44, S D=9.31)$ were recruited for the experiment. None of them had any color-vision disorders. Participants rated the seven emotions (used as referential ratings) before they rated the perceived emotions of expressions based on expressive lights. The order in which the seven expressions were shown was randomized.

\subsection{Results}

Figure 2(a) shows the referential ratings of the seven emotions. $\circ$ indicates the mean valence and arousal values for the emotions, where ellipses represent the standard deviation. In general, our results had a similar distribution to that reported by Russell [11] and Strohmeier et al. [5]. However, our participants rated happy, fear, and anger to be more positive (greater valence value). This might be due to cultural factors as participants from both of their two studies were from Western countries, while our participants were from Eastern countries (Japan).

Figure 2(b) shows the ratings of the expressions based on expressive lights. Mean distances between response emotions ( $\circ)$ and referential emotions $(+)$ are illustrated as line segments (see Table 3 ). It is clearly revealed that participants perceived emotions with quite different levels of valence and arousal compared with the corresponding referential emotions shown in Fig. 2(a). Such results were expected since previous studies suggested that correctly conveying emotions via expressive light cues alone is difficult.

\section{STUDY II: EMOTIONAL EXPRESSION VIA MOTION}

In this study, we explored how people might perceive affect towards in-situ motions. 


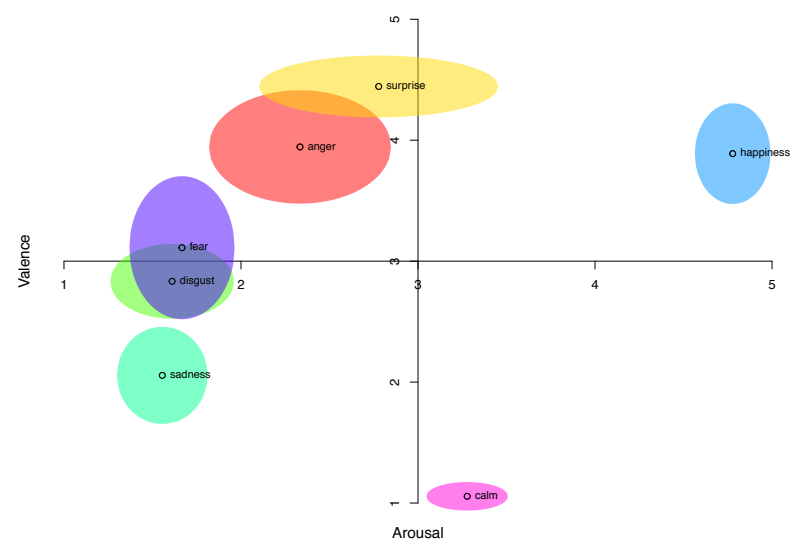

(a)

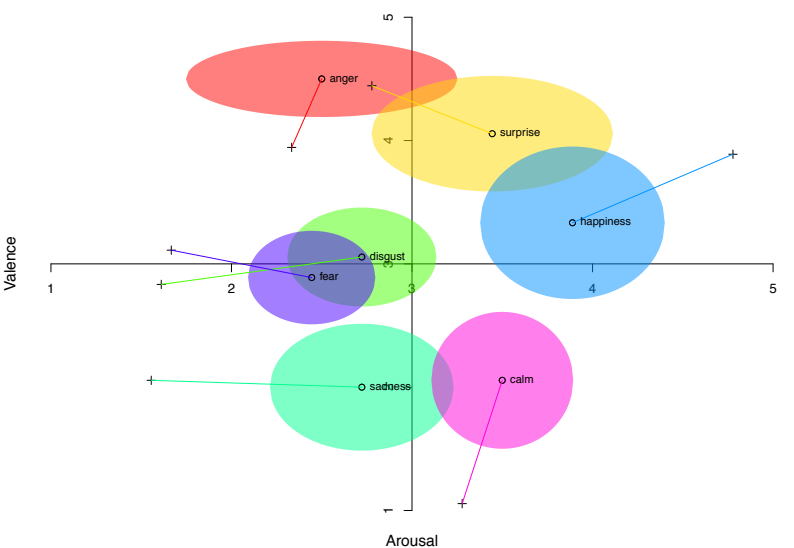

(b)

Figure 2: Circumplex Model of Affect. $\circ$ shows mean valence and arousal ratings of emotions, where ellipses show area encompassing one standard deviation. Figure (a) shows referential ratings of seven emotions. Figure (b) shows ratings of expressions based on expressive lights. Particularly, with regards to Figure (b), line segments show mean distances between response emotions $(\circ)$ and referential emotions $(+)$.

\subsection{Method}

Due to the nature of the Roomba robot, we found two parameters that can well represent the characteristics of its in-situ motions: pattern and speed. To be specific, we designed two in-situ motion patterns, circle and shake, to fit the mobility of the Roomba. For the circle pattern, the robot simply spins 360 degrees clockwise and stops at the initial orientation. For the shake pattern, the robot first turns 45 degrees clockwise and then turns 90 degrees counterclockwise. It then turns 90 degrees clockwise and 90 degrees counterclockwise. Last, it turns 45 degrees clockwise to return to the initial orientation. This pattern design was inspired by both human and animal behaviors. Many people and animals, e.g., dogs, might move similarly in a circle when they have positive feelings and might shake their heads (similar to shake) when they have negative feelings, e.g., expressing disagreement.

We also designed three levels of speed for the robot's in-situ motion: low, medium, and high. According to previous work [4], the use of three levels can sufficiently represent the design space of speed.

\subsection{Participants}

The same eighteen Japanese participants took part in the experiment. They were asked to rate the perceived emotions of expressions based on in-situ motions. The order in which the six expressions were shown was randomized. The experiment had a 2 (pattern: circle vs. shake) $\times 3$ (speed: low, medium, high) within-participant design.

\subsection{Results}

Figure 3 shows the ratings of the expressions based on in-situ motions. The results indicate an interesting relationship between in-situ motion parameters and attribution of affect.

We ran a factorial repeated measures ANOVA to test the ratings for valence and arousal separately. With regards to valence, it showed a significant main effect for both pattern $[F(1,17)=$ $7.59, p<0.05]$ and speed $[F(2,34)=5.72, p<0.05]$. No interaction effect was found. We further conducted post-hoc tests with Holm's correction. The analysis revealed two important points. One, when speed was high, the circle pattern was perceived as significantly more positive than the shake pattern $(p<0.05)$. Two, when the pattern was the circle, the high speed was perceived as significantly more positive than the low speed. Such findings are in line with our hypothesis that humans and animals perform movements similar to the circle pattern to express positive feelings.

With regards to arousal, we found both a significant main effect for pattern $[F(1,17)=5.35, p<0.05]$ and speed $[F(2,34)=$ $76.24, p<0.001]$ and a significant interaction effect $[F(2,34)=$ $5.52, p<0.05]$. This effect might indicate that the shake pattern, compared with the circle one, was particularly strong in conveying emotions with high arousal levels. We conducted post-hoc tests with Holm's correction as well. The findings suggested that, one,

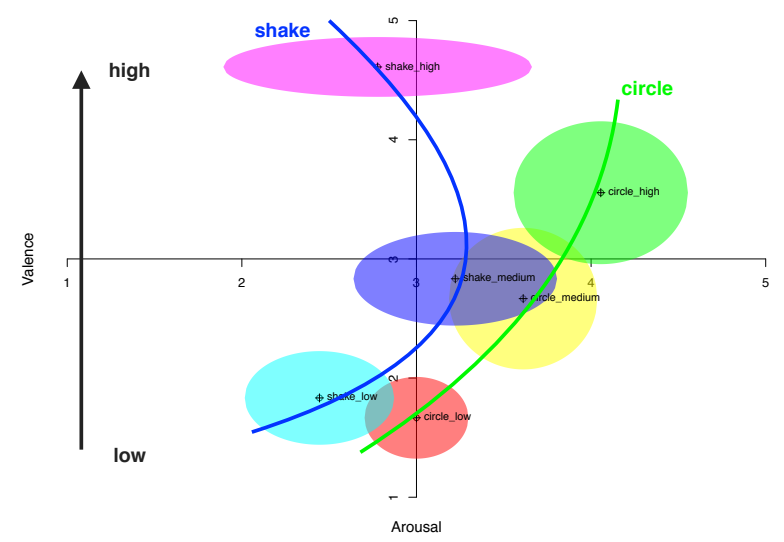

Figure 3: Ratings of expressions based on in-situ motions. 
when the speed was high, the shake pattern was perceived as significantly more intense than the circle pattern $(p<0.05)$, and, two, for both patterns, a higher level of speed was perceived as significantly more intense than a lower level of speed.

\section{STUDY III: COMBINING EXPRESSIVE LIGHTS AND MOTION}

On the basis of the findings from studies I and II, in this study, we investigated whether the robot could better convey emotions by combining in-situ motions and expressive lights.

\subsection{Method}

To evaluate how precisely an expression conveyed a target emotion, we calculated the Euclidian distance between the rating of an expression and its corresponding referential rating. By doing this, we were able to obtain two sets of distances: mean distances between ratings of expressions based on expressive lights alone and the referential ratings and mean distances between ratings of multi-modal expressions and the referential ratings.

The results of study II provided insights into the relationship between in-situ motion characteristics and the attribution of affect. Basically, the speed of motion had a strong and positive relationship with the perceived arousal level. Moreover, the shake pattern was overall perceived to be more negative than the circle pattern, especially when the speed level was high. On the basis of these findings, we hypothesized that multi-modal expressions that appropriately combine in-situ motions and expressive lights were better able to be recognized. In other words, we intuitively assumed that adding appropriate in-situ motions might help to decrease the distances between the ratings of expressions based on expressive lights alone and their corresponding referential ratings. For instance, since the rating for happiness (shown in Figure 2) had both lower valence and arousal levels compared with its referential rating, a reasonable combination was to add in-situ motion with the circle pattern and the high speed level to the corresponding expressive light expressions. Table 2 shows all the combinations for the seven emotions.

\subsection{Participants}

Eleven Japanese in total ( 8 males and 3 females) ranging from 22 to 50 years old $(M=27.91, S D=8.14)$ were recruited for the experiment. None of them had any color-vision disorders. Participants were asked to rate the perceived emotions of expressions based on

Table 2: Parameter settings for design of multi-modal expressions.

\begin{tabular}{c|lcccc}
\hline Emotion & \multicolumn{1}{|c}{ RGB } & \multicolumn{2}{c}{ Period $[m s]$ Waveform } & Pattern & Speed \\
\hline Anger & $255,17,0$ & 896 & square & shake & high \\
Surprise & $255,132,0$ & 747 & square & shake & high \\
Disgust & $255,0,179$ & 1645 & square & shake & low \\
Sadness & $21,0,255$ & 3310 & sinusoid & shake & low \\
Happiness & $255,157,0$ & 1123 & square & circle & high \\
Fear & $166,0,255$ & 1377 & square & shake & high \\
Calm & $255,255,255$ & 2000 & sinusoid & circle & low \\
\hline
\end{tabular}

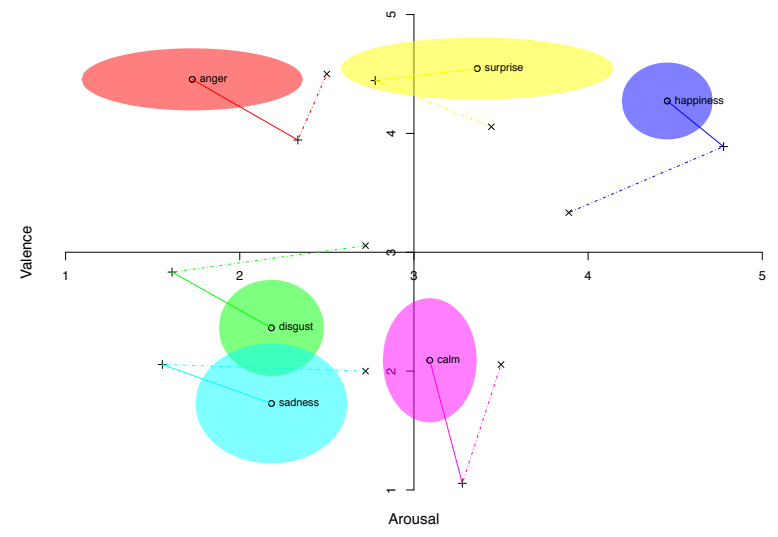

Figure 4: Ratings of multi-modal expressions that combine in-situ motions and expressive lights. $\odot$ indicates mean valence and arousal values for ratings of multi-modal expressions, $x$ indicates mean valence and arousal values for ratings of expressions based on expressive lights alone, and + indicates referential ratings.

multi-modal expressions that combine in-situ motions and expressive lights. The order in which six expressions were shown was randomized (due to technical problems during the experiment, we failed to show the expression for fear).

\subsection{Results}

Figure 4 shows the ratings of the expressions. $\circ$ indicates the mean valence and arousal values for the ratings of the multi-modal expressions, $\times$ indicates the mean valence and arousal values for the ratings of the expressions based on expressive lights alone, and + indicates referential ratings. Mean distances between response emotions ( $(\circ)$ and referential emotions (+) are illustrated as line segments, and mean distances between response emotions $(\times)$ and referential emotions $(+)$ are shown as dashed line segments.

Table 3 shows the mean distances (computed in the Circumplex Model) of emotion attributes in studies I and study III. The column Distance_I represents the mean distances between the referential ratings of emotions and the ratings of the expressions based on expressive lights alone. Distance_III represents the mean distances between the referential ratings and the ratings of the multi-modal expressions that combine in-situ motions and expressive lights. Difference demonstrates the differences between Distance_I and Distance_III (Distance_I - Distance_III). Each difference reflects how much an added in-situ motion helped a corresponding expressive light expression with conveying a target emotion. A minus value of difference indicates that the in-situ motion had a positive contribution to the expression of a target emotion, and a larger value suggests a greater amount of contribution.

\section{DISCUSSION}

In general, the experimental results show strong support for our three hypotheses (see 3.4). Study I was done to re-examine designs for emotions expressed via expressive lights. The findings reveal 
Table 3: Mean distances in Circumplex Model of emotion attributes in studies I and III.

\begin{tabular}{c|ccc}
\hline Emotion & Distance_I & Distance_III & Difference \\
\hline Anger & 0.58 & 0.79 & 0.21 \\
Surprise & 0.77 & 0.59 & $\mathbf{- 0 . 1 8}$ \\
Disgust & 1.13 & 0.74 & $\mathbf{- 0 . 3 9}$ \\
Sadness & 1.17 & 0.71 & $\mathbf{- 0 . 4 6}$ \\
Happiness & 1.05 & 0.50 & $\mathbf{- 0 . 5 4}$ \\
Calm & 1.02 & 1.05 & 0.03 \\
\hline
\end{tabular}

that expressions based on expressive lights alone are not able to convey target emotions precisely. The distances between the ratings of response emotions (expressed via expressive lights) and ratings of referential emotions were quite large. Study II was done to explore emotions expressed via in-situ motions. The results suggest a strong relationship between in-situ motion parameters, pattern and speed, and perceived affect. Specifically, the speed factor contributed heavily to the intensity (arousal level) of an emotion, while the pattern factor contributed to the perceived valence level of an emotion. Study III was done to investigate multi-modal expressions that combine in-situ motions and expressive lights. The results show that adding in-situ motion to expressive lights improves affective expression for some emotions but not all.

We found that an in-situ motion seems to act as an "amplifier" to its corresponding expressive lights. For instance, when an insitu motion with the circle pattern and high level of speed was added to the expressive light expression for happiness (see Table 2), the ratings for this emotion had both higher levels of valence and arousal, and the mean distance between the response emotion and referential emotion was decreased by 0.54 (see Figure 4 and Table 3). Similarly, when an in-situ motion with the shake pattern and low level of speed was added to the expressive light expression for sadness (see Table 2), the ratings for this emotion had both lower levels of valence and arousal, and the mean distance was decreased by 0.46 (see Figure 4 and Table 3). However, the amplifier effect might have negatively influenced the emotional expression for some emotions. For instance, when an in-situ motion with the shake pattern and high level of speed was added to the expressive light expression for anger (see Table 2), the ratings for this emotion had a lower valence and higher arousal, but the mean distance was increased by 0.21 (see Figure 4 and Table 3). In other words, the in-situ motion with the shake pattern and high level of speed made the perceived anger emotion too negative.

\subsection{Limitations}

Our findings were mainly limited in terms of three points. To combine in-situ motions with expressive lights, we assumed that these two modalities were independent of each other. In other words, we ignored that there might be interaction effects between the two modalities. Although our results suggest an improvement in emotion recognition for most emotions, future work still needs to be done to carefully investigate such interaction effects. A second limitation is that we designed only six expressions ( 2 patterns $\times 3$ speed levels) for testing the in-situ motions. In study II, we successfully discovered the relationship between in-situ motion characteristics and attribution of affect based on the six expressions. However, the findings were not sufficient enough to give any suggestion on how to fine-tune the in-situ motions (speed in particular) to make optimal multi-modal expressions. A third limitation is that, in this work, we did not test gender effects since people of different sexes might have different levels of sensitivity with regards to emotional perception. In their work, Saerbeck and Bartneck [4] found no significant effects or significant interactions for gender on any combination of their motion characteristics. Therefore, we might assume that there is also no significant effects for gender on any combination of our in-situ motion characteristics. However, there might still be gender effects on the perception of expressive lights, especially on the color factor. We suggest that future work need to take the three limitations into account to achieve more precise design guidelines for designing emotional expressions via in-situ motion cues and expressive light cues.

\section{ACKNOWLEDGMENTS}

This study was partially supported by JSPS KAKENHI "Cognitive Interaction Design” (No. 26118005).

\section{REFERENCES}

[1] Cindy L. Bethel and Robin R. Murphy. 2008. Survey of non-facial/non-verbal affective expressions for appearance-constrained robots. IEEE Transactions on Systems, Man, and Cybernetics, Part C (Applications and Reviews) 38, 1 (Jan. 2008), 83-92. https://doi.org/10.1109/TSMCC.2007.905845

[2] Thomas Fincannon, Laura Barnes, Murphy Robin R., and Dawn Riddle. 2004. Evidence of the need for social intelligence in rescue robots. In Proceedings of the 14th IEEE/RSJ International Conference on Intelligent Robots and Systems, vol. 2. 1089-1095. https://doi.org/10.1109/IROS.2004.1389542

[3] Robin R. Murphy, Dawn Riddle, and Eric Rasmussen. 2004. Robot-assisted medical reachback: a survey of how medical personnel expect to interact with rescue robots. In Proceedings of the 13th IEEE International Workshop on Robot and Human Interactive Communication. 301-306. https://doi.org/10.1109/ROMAN. 2004.1374777

[4] Martin Saerbeck and Christoph Bartneck. 2010. Perception of affect elicited by robot motion. In Proceedings of the 5th ACM/IEEE International Conference on Human-Robot Interaction. 53-60. https://doi.org/10.1109/HRI.2010.5453269

[5] Paul Strohmeier, Juan Pablo Carrascal, Bernard Cheng, Margaret Meban, and Roel Vertegaal. 2016. An evaluation of shape changes for conveying emotions. In Proceedings of the $2016 \mathrm{CHI}$ Conference on Human Factors in Computing Systems (CHI '16). ACM, New York, NY, USA, 3781-3792. DOI: https://doi.org/10.1145/2858036.2858537

[6] Jessica Rebecca Cauchard, Kevin Y. Zhai, Marco Spadafora, and James A. Landay. 2016. Emotion encoding in human-drone interaction. In The Eleventh ACM/IEEE International Conference on Human Robot Interaction. IEEE Press, Piscataway, NJ, USA, 263-270. http://dl.acm.org/citation.cfm?id=2906831.2906878

[7] Patrice D. Tremoulet and Jacob Feldman. Perception of animacy from the motion of a single object. Perception 29.8 (2000): 943-951.

[8] Kazunori Terada, Atsushi Yamauchi, and Akira Ito. 2012. Artificial emotion expression for a robot by dynamic color change. In Proceedings of the 21st IEEE International Symposium on Robot and Human Interactive Communication. 314-321. https://doi.org/10.1109/ROMAN.2012.6343772

[9] Sichao Song and Seiji Yamada. 2017. Expressing emotions through color, sound, and vibration with an appearance-constrained social robot. In Proceedings of the 2017 ACM/IEEE International Conference on Human-Robot Interaction (HRI '17). ACM, New York, NY, USA, 2-11. DOI: https://doi.org/10.1145/2909824.3020239

[10] Andrew J. Elliot, Mark D. Fairchild, and Anna Franklin. 2015. Handbook of Color Psychology. Cambridge University Press.

[11] James A. Russell. 1980. A circumplex model of affect. Journal of Personality and Social Psychology 39, 6, 1161.

[12] Peter J. Lang, Margaret M. Bradley, and Bruce N. Cuthbert. 2008. International affective picture system (IAPS): Affective ratings of pictures and instruction manual. Technical Report A-8.

[13] Paul Ekman and Wallace V. Friesen. 1975. Unmasking the face: A guide to recognizing emotions from facial cues.

[14] Tadas Baltrušaitis, Laurel D. Riek, and Peter Robinson. 2010. Synthesizing expressions using facial feature point tracking: How emotion is conveyed. In Proceedings of the 3rd International Workshop on Affective Interaction 
in Natural Environments (AFFINE '10). ACM, New York, NY, USA, 27-32. DOI=http://dx.doi.org/10.1145/1877826.1877835

[15] Lola D. Canamero and Jakob Fredslund. I show you how I like you - can you read it in my face? In IEEE Transactions on Systems, Man, and Cybernetics - Part A: Systems and Humans, vol. 31, no. 5, pp. 454-459, Sep 2001. doi: 10.1109/3468.952719

[16] Daniel J. Rea, James E. Young, and Pourang Irani. 2012. The Roomba mood ring: an ambient-display robot. In Proceedings of the seventh annual ACM/IEEE international conference on Human-Robot Interaction (HRI '12). ACM, New York, NY, USA, 217-218. DOI: https://doi.org/10.1145/2157689.2157763
[17] D. S. Syrdal, K. L. Koay, M. Gácsi, M. L. Walters and K. Dautenhahn, "Video prototyping of dog-inspired non-verbal affective communication for an appearance constrained robot," 19th International Symposium in Robot and Human Interactive Communication, Viareggio, 2010, pp. 632-637. doi: 10.1109/ROMAN.2010.5598693

[18] M. Häring, N. Bee and E. André, "Creation and Evaluation of emotion expression with body movement, sound and eye color for humanoid robots," 2011 RO-MAN, Atlanta, GA, 2011, pp. 204-209. doi: 10.1109/ROMAN.2011.6005263

[19] Diana Löffler, Nina Schmidt, and Robert Tscharn. 2018. Multimodal Expression of Artificial Emotion in Social Robots Using Color, Motion and Sound. In Proceedings of the 2018 ACM/IEEE International Conference on Human-Robot Interaction (HRI '18). ACM, New York, NY, USA, 334-343. DOI: https://doi.org/10.1145/3171221.3171261 